\title{
Fullerene dissymmetrization as a means to achieve single enantiomer electron acceptors with maximized chiroptical responsiveness
}

Wenda Shi ${ }^{a}$, Francesco Salerno ${ }^{a, c}$, Alejandro Santana Bonilla ${ }^{a}$, Matthew D. Ward ${ }^{b, c}$, Xueyan Hou ${ }^{d}$, T. John S. Dennis ${ }^{d}$, Alasdair J. Campbell ${ }^{b, c}$, Kim E. Jelfs ${ }^{a, c}$ and Matthew J. Fuchter ${ }^{a, c^{*}}$

${ }^{a}$ Department of Chemistry and Molecular Sciences Research Hub, Imperial College London, White City Campus, 80 Wood Lane, London W12 0BZ, UK, ${ }^{b}$ Department of Physics, Imperial College London, South Kensington Campus, London SW7 2AZ, UK, ${ }^{c}$ Centre for Processable Electronics, Imperial College London, South Kensington Campus, London SW7 2AZ, UK, ${ }^{d}$ School of Physics and Astronomy and Materials Research Institute, Queen Mary University of London, Mile End Road, London E1 4NS, UK, *e-mail: m.fuchter@imperial.ac.uk

Keywords: chiral materials, chiroptical, fullerenes, organic field-effect transistors, circularly polarized light

\footnotetext{
Abstract

Solubilized fullerene derivatives have revolutionised the development of organic photovoltaic devices, acting as excellent electron acceptors. The addition of solubilizing addends to the fullerene cage results in a large number of isomers, which are generally employed as isomeric mixtures. Moreover, a significant number of these isomers are chiral, which further adds to the isomeric complexity. The opportunities presented by single isomer, and particularly single enantiomer, fullerenes in organic electronic materials and devices are poorly understood. Here we separate 10
} 
pairs of enantiomers from the 19 structural isomers of bis[60]PCBM, using them to elucidate important chiroptical structure-property relationships and demonstrating their application to a single enantiomer circularly polarized (CP) light detecting device. We find that larger chiroptical responses occur through inherent chirality of the fullerene cage and particularly through transitions with low $\mathrm{CT}$ character. When used in a single enantiomer organic field-effect transistor device, we demonstrate the potential to discriminate CP light with a fast light response time and with a very high photocurrent dissymmetry factor $\left(g_{p h}= \pm 1.35\right)$. Our study thus provides key strategies to design fullerenes with large chiroptical responses for use as single enantiomer components of organic electronic devices. We anticipate that our data will position chiral fullerenes as an exciting material class for the growing field of chiral electronic technologies.

\section{Introduction}

Fullerene derivatives have played a hugely important role in the development of organic electronic devices, particularly organic solar cells. They remain the most widely used electron acceptors in organic/hybrid photovoltaic devices $(\mathrm{OPVs}),{ }^{[1-4]}$ while also demonstrating excellent photo responsivity as broadband photodetectors. ${ }^{[5-10]}$ Parent fullerene structures have extremely low solubility, which limits their processability and miscibility with host materials in bulk heterojunction OPVs; an issue which has been addressed through the production of more soluble derivatives using simple cycloaddition chemistry. Bis[60]PCBM (bis(1-[3-(methoxycarbonyl)propyl]-1-phenyl)$\left.[6,6] \mathrm{C}_{62}\right)$ is one such derivative and one of the most utilized solubilized fullerenes, which is easy to synthesize, highly processable, and solar cells based on this material have high power conversion efficiencies. ${ }^{[11-18]}$ The addition of two solubilizing addends to $\mathrm{C}_{60}$ results in a large number of structural isomers however, each with varying energetic properties (influencing device voltage) and 
morphological properties (influencing device current). Despite this, bis[60]PCBM is synthesized and used as an isomeric mixture, and the role of individual isomers on morphological, spectroscopic and device performance is very poorly understood. In a previous study, Dennis and co-workers separated the 19 structural isomers of bis[60]PCBM and characterized them using a combination of NMR, UVvis and retention times by HPLC. ${ }^{[19,20]}$ Although the performance of a single structural isomer bis[60]PCBM in organic devices was not investigated, isomerically-pure fullerene derivatives have generally been reported to exhibit higher power conversion efficiency (PCE) than the corresponding isomeric mixture. ${ }^{[21,22]}$ This implies that the further study of single bis[60]PCBM isomers may hold significant promise.

The complex isomeric composition of bis[60]PCBM is not limited to structural isomers however. Of the synthetically accessible 19 bis[60]PCBM isomers, 13 are chiral, which gives rise to 13 pairs of enantiomers (see Figure 1). It is interesting to consider what opportunities could emerge from access to enantiomerically-pure fullerenes. To date, chiral fullerene derivatives have mostly been explored as an academic curiosity and in limited applications, with a notable exception perhaps being enantioselective catalysis. ${ }^{[23]}$ Given the importance of this material class to organic electronic devices, it is curious that single enantiomer fullerene devices are yet to be explored in this context. This may be because the opportunities provided by chiral organic semiconducting materials are, in general, underexploited in technological applications. ${ }^{[24]}$ Nonetheless, it is likely that chiral composition dependent effects on device performance would be observed, ${ }^{[25,26]}$ as would the potential to render fullerene devices sensitive to circularly polarized (CP) light, through preferential absorption of left or right-handed CP light (circular dichroism, CD). Such CP functionality would potentially have far- 
reaching applications, including in enantioselective sensing, optical communication, and quantum computation. ${ }^{[27-34]}$

In particular, chiral conjugated materials with excellent CD have strong potential in the development of CP light detectors, either transistors or diodes. ${ }^{[35-37]}$ The first CP light sensitive photodiode was developed by Meskers and co-workers in 2010, utilising a polyfluorene with chiral side chains. ${ }^{[35]}$ In 2013, Fuchter, Campbell and co-workers demonstrated the first (and currently only) CP light sensitive phototransistor (CP-photoFET), using a chiral small molecule helicene. ${ }^{[36]}$ Other approaches recently reported for CP detection in devices include self-assembled small molecules, metamaterials, and perovskite hybrid materials. ${ }^{[37-42]}$ Despite these emerging approaches that use an array of material classes, organic molecules remain highly attractive for the development of CP light photodetectors, due to their tuneable optical characteristics, mechanical flexibility, low processing cost and scalability (through printing, etc).

In this work, we separate and systematically study 10 pairs of enantiomers from the 19 structural isomers of bis[60]PCBM. We analyse the chiroptical responses of these isomers and elucidate the origin of larger absorption dissymmetry ( $g_{a b s}$ factors) in some isomers over others. Specifically, we show that larger chiroptical responses occur in cases with greater asymmetry of the electron distribution within the fullerene cage. We showcase the application of our single enantiomer bis[60]PCBM materials in the production of a single enantiomer CP-photoFET device, with a very high photocurrent dissymmetry factor $\left(g_{p h}= \pm 1.35\right)$. Thus, our study determines a clear set of design principles to generate chiral fullerene derivatives with maximised chiroptical activity, and with significant potential in the burgeoning field of chiral (nano)technology and devices. 


\section{Results and Discussion}

For bridged $\mathrm{C}_{60}$ monoadducts, such as $\mathrm{PCBM}$, there are 8 remaining non-equivalent double bonds susceptible to the addition of a second addend. Formation of the bisadduct therefore gives rise to 8 regioisomers: Cis-1, Cis-2, Cis-3, E, Trans-1, Trans-2, Trans-3 and Trans-4 (Figure 1). ${ }^{[43-45]}$ Bond type naming is defined by the relative position of the second addend with respect to the first. When both addends are found on the same hemisphere, the isomer is named Cis, if on the opposite hemisphere, Trans, and if on the equatorial plane, $E$. This naming system also reflects the electronic properties of the isomers, where structural isomers of the same bond type have similar UV-Vis absorption spectra. ${ }^{[19,43]}$ Here, we only consider 6 bond types since the $C i s-1$ isomer is not formed during bis[60]PCBM synthesis in any appreciable amount due to steric effects and the Trans-1 isomer is not chiral due to the presence of a mirror plane (Figure 1). Of the structural isomers contained within these 6 remaining bond type groups, 13 pairs of enantiomers can be theoretically predicted according to their symmetry properties. ${ }^{[20]}$

In general terms, fullerene molecules can be chiral by virtue of a chiral carbon cage (for example, in higher fullerenes, such as $\left.\mathrm{C}_{76}, \mathrm{C}_{84}\right)$, stereogenic elements in the addends, or from the geometric arrangement of the addends via a chiral addition pattern. In the case of the bis[60]PCBM derivative employed in this study - the one principally used in organic electronic devices, as for others $\mathrm{C}_{60}$ bisadducts previously reported in literature, ${ }^{[46,47]}$ some structural isomers are inherently chiral while others are non-inherently chiral. In the former case, the isomers are chiral due to the desymmetrization of the $\mathrm{C}_{60}$ cage by the particular addition pattern, regardless of the nature of the addends. In the latter case, the dissymmetry arises exclusively from the stereogenic units of the 
addends. $^{[46]}$ In a previous study, Dennis and co-workers named the structural isomers of bis[60]PCBM based on the location of the second addend and the symmetry, e.g. $\left(\mathrm{C}_{1}\right) 21,40$ bis[60]PCBM. ${ }^{[20]}$ Here, we adapt that nomenclature to explicitly include descriptors of fullerene chirality and the stereochemistry of the addends, according to the systematic system devised by Diederich and Thilgen. ${ }^{[46]}$ The assigned systematic names are given in Figure $\mathbf{1}$ and the nomenclature system is further described in the SI.

The 19 structural isomers were isolated using the previously reported method and identified by UVVis, ${ }^{13} \mathrm{C}$ NMR and retention time (i.e. polarity). ${ }^{[19,20]}$ The 13 chiral isomers of bis[60]PCBM, obtained as racemates, were subjected to further purification via peak-recycling HPLC using chiral columns (Chiralpak IE and IF). 10 out of 13 pairs of enantiomers were successfully resolved and purified to $>99 \%$, which represents at least one enantiomeric pair for every bond type isomer. This isomeric series provides the means to systematically study the chiroptical response of the chiral fullerene isomers as a function of substitution pattern and symmetry for the first time.

To assign the absolute configuration of the isolated bis[60]PCBM stereoisomers, we compared experimentally obtained CD spectra with the simulated spectra. Such an approach is increasingly common for assignment of the absolute configuration and has been applied previously to chiral fullerenes. ${ }^{[48]}$ The bis[60]PCBM isomeric structures were optimized at the B3LYP/6-31G(d) level. Under the assumption that the extended butyric acid addend substituent will have little impact on the chiroptical properties of the fullerene, this moiety was truncated to a methyl group for all the computational structures under study. The excellent correlation of experimental and calculated CD spectra later confirmed this assumption. UV-Vis and CD spectra were calculated via time-dependent 
density functional theory (TD-DFT) for the first 50 excited states at the B3LYP/6-31G(d) level of theory and compared to experimental spectra using SpecDis. ${ }^{[49]}$ A similarity factor higher than 0.8 was obtained for all of the 10 isomers. Therefore, the absolute configuration of the enantiomeric pairs obtained could be assigned with high degree of confidence as shown in Table S1. All spectra and assignments can be found in SI.

Due to the high number of $\pi \rightarrow \pi^{*}$ transitions located in the UV-Vis region (see SI for full set of UVVis and CD spectra), very spectroscopically rich CD spectra are observed (Figure S4). As would be expected, all of the isolated pairs of enantiomers show an equal and opposite CD response. Importantly, diastereomers belonging to the same bond type (Cis-2, Cis-3, Trans-2, etc.) display almost identical CD spectra, with only minor differences in amplitude and excitation energy, that can be therefore considered an inherent stereochemical fingerprint of each addition pattern of these bis[60]PCBM isomers. Addend substituent stereochemistry appears therefore to have a negligible impact on the chiroptical properties, as has been previously observed in specific examples of similar systems. ${ }^{[50]}$ From an application point of view, the variety of CD responses contained within this series allows access to a CP-light responsivity across a large region of the UV-vis spectrum of potential usage in CP-light detecting devices.

To further investigate how the chiroptical properties observed relate to the isomeric structures of this series, the experimental absorption dissymmetry factor $g_{\text {abs }}=\Delta \varepsilon / \varepsilon$ was analysed. This value provides a dimensionless measure of the chiroptical response of a given molecule, normalised by absorption. The $g_{\text {abs }}$ factors of all the stereoisomers, ordered by bond type, are shown in Figure 2a for representative enantiomers. The magnitude of $g_{a b s}$ varies by approximately two orders of magnitude 
as a function of stereoisomeric series, from $10^{-4}-10^{-3}$ (typical of chiral organic small molecules) up to the value of $3 \times 10^{-2}$. Values of this order of magnitude are highly uncommon for an isolated molecule in solution. ${ }^{[51]}$ Moreover, a particularly large chiroptical response occurs at $\sim 600 \mathrm{~nm}$, a longer wavelength than the one typically achievable with other conjugated chiral small molecules, such as helicenes, biraryls and cyclophanes. ${ }^{[51]}$ Theoretically calculated $g_{\text {abs }}$ at the B3LYP/6-31G(d) level of theory are of the same order magnitude, therefore in good agreement, with the experimental values (both experimental and simulated values at the peaks maxima are listed in Table S3).

The six bond types can be further divided into two groups according to their $g_{\text {abs }}$ values. Stereoisomers from $C i s-2, E$ and Trans-4 show a relatively low $g_{\text {abs }}$ value $\left(\sim 10^{-3}\right)$, while stereoisomers from $C i s-3$, Trans-2 and Trans-3 show a $g_{\text {abs }}$ value of up to one order of magnitude higher $\left(\sim 10^{-2}\right)$. This significative difference in chiroptical response can be qualitatively rationalized using symmetrybased arguments. In the group of isomers with lower $g_{\text {abs }}$, the addition pattern on the $\mathrm{C}_{60}$ cage (without considering the nature of the addends) contains a mirror plane as shown in Figure 2b, upper row. As described above, bis[60]PCBM isomers of these bond type groups (Cis-2, E and Trans-4) are noninherently chiral; that is the chirality arises only by virtue of the stereogenic centres on the addends. This is equivalent to stating that the chromophore is fundamentally achiral and the chiroptical response derives only from perturbation of the substituents. The group of isomers with higher $g_{\text {abs, }}$ instead, have addition patterns with $\mathrm{C}_{2}$ symmetry (Cis-3, Trans-2 and Trans-3); these bis[60]PCBM isomers are therefore said to be inherently chiral. This means the fullerene chromophore is inherently chiral and therefore the chiroptical response would be expected to increase. Ultimately, this difference in symmetry determines the distortion of $\pi$-electron distribution in the cage, with achiral addition patterns leading to more uniform electron distributions than those that are chiral. 
TD-DFT results were further analysed using the software TheoDORE, which allows the study of the nature of the excited states in greater detail by employing the Natural Transition Orbital (NTO) formalism. ${ }^{[52]}$ For a given excitation, NTOs can be associated to a hole-electron pair, making it easier to visualize the movement of charges across different molecular fragments. This enables one to assess the role of charge-transfer $(\mathrm{CT})$ character in the $\pi \rightarrow \pi^{*}$ transitions of different isomers and how this relates to their chiroptical properties. ${ }^{[53]}$ We observe that excited states with high rotatory strength $(R)$ are generally states with low CT character. This is more clearly observed for inherently chiral substitution patterns (Cis-2, Trans-2, Trans-3) as shown in Figure S9. In Figure 2c, we show part of the analysis for the $E$ and Trans-2 isomers (full analysis can be found in SI). In both cases, for the states with the highest charge-transfer character, the transition occurs as a movement of $\pi$-electrons from the $\mathrm{C}_{60}$ to the addends. In contrast, the states with highest rotatory strength (state 25 for $E$ and state 8 for Trans-2) are described by a strong rotational movement of $\pi$-electrons solely on the surface of the cage with very low CT character (weak translation of charges). It is well known that the $g$ factor of a chiral chromophore, is defined by equation (1):[54]

$$
g=\frac{4|\mathbf{m}| \cdot|\boldsymbol{\mu}| \cdot \cos \theta}{|\mathbf{m}|^{2}+|\boldsymbol{\mu}|^{2}}
$$

Where $m$ is the magnetic transition dipole of the chromophore, $\mu$ is the electric transition dipole and $\theta$ is the angle between $m$ and $\mu$.

We observe that the magnetic transition dipole moment is significantly higher in magnitude than the electric transition dipole for those excited states with high rotatory strength. Moreover, these states are characterized by a very small angle $\theta$ when the chromophore is chiral, implying a strong coupling 
between $m$ and $\mu$. This likely accounts for the higher order of magnitude of $g_{\text {abs }}$ observed in those isomers with inherently chiral substitution pattern. The distance between the addends seems not to play a crucial role since states with high rotatory strength are mainly local and do not involve charge delocalization onto the addends.

With access to single stereoisomer bis[60]PCBM molecules, we sought to showcase their application in the context of an organic electronic device. The Equatorial structural isomer is the most abundant within our pre-separated isomeric mixture, representing around $15.6 \%$ of the mass balance. ${ }^{[19]}$ This isomer was therefore selected for a proof of concept device, given the ability to access suitable quantities for device fabrication. Enantiomers (anti,S)16,17-bis[60]PCBM and (anti,R)16,17bis[60]PCBM were separately used to build bottom-gate, bottom-contact organic field-effect transitiors (OFETs) on prepatterned substrates. The output curve of (anti,S)16,17-bis[60]PCBM as a n-type material produced well-behaved linear and saturated regions as shown in Figure 3e. The transfer characteristics in the dark (Figure 3a) showed a turn-on voltage of $40 \mathrm{~V}$, a low off-current $\left(1 \times 10^{-11} \mathrm{~A}\right)$, an on/off ratio of $10^{3}$, and a saturation electron mobility $3.7 \times 10^{-4} \mathrm{~cm}^{2} \mathrm{~V}^{-1} \mathrm{~s}^{-1}$. To the best of our knowledge, this represents the first OFET prepared from a single isomer bis[60]PCBM material. The performance of these devices could be further optimised through alternative device architecture, fabrication procedure, etc. Both enantiomers exhibit analogous OFET behaviour, as would be expected (Figure 3b).

The use of an enantiomerically-pure semiconductor enables the photoresponse of OFETs to be sensitive to the circular polarization of light. While there are a number of emerging material and device approaches to CP-light detection, only one CP-photoFET has been previously reported, by 
Fuchter, Campbell and co-workers, using an enantiomerically pure helicene. ${ }^{[36]}$ To study the CP light responsivity of the enantiopure fullerene-based transistors, single enantiomer OFETs were illuminated using a laser diode at $405 \mathrm{~nm}$, which corresponds to one of the more intense CD peaks. The transfer characteristics in the absence and under left handed CPL, right handed CPL and unpolarized light are shown in Figure 3a,b. Devices fabricated using opposite enantiomers displayed opposite responsiveness to the CP light of the same handedness, as well as the same response to unpolarized light. The response of the devices to unpolarized light occurs in between the response for CP light of opposite handedness, in agreement with the results from perovskite chiral photodetecting devices. ${ }^{[40]}$ The off currents of both devices increase by around two orders of magnitude, from $10^{-11}$ to $10^{-9} \mathrm{~A}$, and the turn-on voltages shift from 40 to $20 \mathrm{~V}$ when exposed to light. From this data, it is possible to extract a $g$ factor for the photocurrent generation $\left(g_{p h}\right)$, which is up to an impressive 1.35 (Figure 3a,b). ${ }^{[54]}$ This large and selective CP photoresponse is of high importance for CP-light detectors and suggests significant promise for enantiomerically pure fullerene isomers in such an application. We note that $g_{p h}$ is significantly larger than $g_{a b s}$ (at $405 \mathrm{~nm}$ ). This outcome is consistent with the previously reported helicene CP-photoFET. ${ }^{[36]}$ We propose that the enhancement of $g_{p h}$ relative to $\mathrm{g}_{\mathrm{ph}}$ arises from two cooperative device mechanisms: the $\mathrm{CP}$ selective photogeneration of electrons in the channel, which increases the majority carrier density and the $\mathrm{CP}$ selective photogeneration of holes, which accumulate at the source electrode, reducing the barrier to electron injection. This model is described in full in the Supporting Information.

The light intensity and time dependent CP photoresponse of the chiral fullerene-based devices was also explored. These CP photoFETs were very sensitive to light even at low intensity of illumination $0.06 \mathrm{~mW} \mathrm{~cm}^{-2}$, where an increase of the off current of 1.4 times was observed compared to that in the 
dark Figure 3c. The steady state photocurrent gradually increased and did not reach the saturation from 0.06 to $18.9 \mathrm{~mW} \mathrm{~cm}^{-2}$. The time-dependent photoresponse shows fast symmetric rise time and decay time with sharp square shape as well as an obvious dissymmetric CP response shown in Figure 3f, a substantial improvement and better reversibility over the long decay seen in the helicene CPphotoFETs. ${ }^{[36]}$ The rise time is around $22.5 \mathrm{~ms}$ and the fall time is $25.1 \mathrm{~ms}$ Figure S5, which is much shorter than in [60]PCBM single crystal photodetector (350-500 ms). ${ }^{[9]}$ Therefore, a device with such a fast light response could in principle be applied to detect higher frequency CP light without delay for information transmission.

\section{Conclusion}

We successfully separated and assigned the absolute configurations to 10 pairs (6 bond types) of bis[60]PCBM enantiomers, one of the most widely used fullerene bisadducts for optoelectronic devices. The broadband UV-visible-NIR chiroptical response indicates great potential in diverse applications and the possibility of tuning device detection wavelength by employing different isomers. We fabricated the first photoFET based on enantiomerically pure fullerene and showed it was able to discriminate circularly polarized light in a highly effective manner. The device gave an excellent $g_{p h}( \pm 1.35)$ and fast response. Such promising properties can be further applied in CP OPD and other CP photodetecting technologies. For example, the use of patterning techniques - available for solution-processable organic semiconductors - should allow the creation of arrays of circularly polarized light-sensitive devices, as well as integration with CMOS electronics. Overall, this study further develops chiral organic materials for CP light detection, positioning enantiopure fullerenes as an exciting material class in such chiral technologies. 


\section{Supporting Information}

Electronic Supporting Information file is available from the Wiley Online Library or from the authors.

\section{Acknowledgements}

We would like to thank the Engineering and Physical Science Research Council for funding (EP/L016702/1, EP/R00188X/1). We would also like to thank Prof. Hanying Li and Zhejiang university for providing funding for WS to conduct research in Imperial College. KEJ acknowledges the Royal Society for a University Research Fellowship and the ERC for funding via grant agreement no. 758370 (ERC-StG-PE5-CoMMaD).

\section{Competing financial interests}

None 


\section{References}

[1] G. Yu, J. Gao, J. C. Hummelen, F. Wudl, A. J. Heeger, Science 1995, 270, 1789.

[2] A. B. Sieval, J. C. Hummelen, in Organic Photovoltaics, Wiley-VCH Verlag GmbH \& Co. KGaA, Weinheim, Germany, 2014, pp. 209-238.

[3] C. H. Chiang, C. G. Wu, Nature Photonics 2016, 10, 196.

[4] R. Ganesamoorthy, G. Sathiyan, P. Sakthivel, Solar Energy Materials and Solar Cells 2017, $161,102$.

[5] Z. Tang, Z. Ma, A. Sánchez-Díaz, S. Ullbrich, Y. Liu, B. Siegmund, A. Mischok, K. Leo, M. Campoy-Quiles, W. Li, K. Vandewal, Advanced Materials 2017, 29, 1702184.

[6] K. Szendrei, F. Cordella, M. v. Kovalenko, M. Böberl, G. Hesser, M. Yarema, D. Jarzab, O. v. Mikhnenko, A. Gocalinska, M. Saba, F. Quochi, A. Mura, G. Bongiovanni, P. W. M. Blom, W. Heiss, M. A. Loi, Advanced Materials 2009, 21, 683.

[7] X. Zhao, T. Liu, H. Liu, S. Wang, X. Li, Y. Zhang, X. Hou, Z. Liu, W. Shi, T. J. S. Dennis, ACS Applied Materials and Interfaces 2018, 10, 42715.

[8] X. Zhao, T. Liu, Y. Cui, X. Hou, Z. Liu, X. Dai, J. Kong, W. Shi, T. J. S. Dennis, Nanoscale 2018, 10, 8170 .

[9] X. Zhao, T. Liu, X. Hou, Z. Liu, W. Shi, T. J. S. Dennis, Journal of Materials Chemistry C 2018, 6, 5489.

[10] X. Zhao, T. Liu, W. Shi, X. Hou, Z. Liu, T. J. S. Dennis, Journal of Physical Chemistry C 2018, 122, 8822 .

[11] J. Yan, B. R. Saunders, RSC Advances 2014, 4, 43286.

[12] M. Lenes, G.-J. A. H. Wetzelaer, F. B. Kooistra, S. C. Veenstra, J. C. Hummelen, P. W. M. Blom, Advanced Materials 2008, 20, 2116.

[13] J. Nelson, Materials Today 2011, 14, 462.

[14] M. Lenes, S. W. Shelton, A. B. Sieval, D. F. Kronholm, J. C. (Kees) Hummelen, P. W. M. Blom, Advanced Functional Materials 2009, 19, 3002.

[15] M. H. Yun, G. H. Kim, C. Yang, J. Y. Kim, Journal of Materials Chemistry 2010, 20, 7710.

[16] L. Ye, S. Zhang, D. Qian, Q. Wang, J. Hou, Journal of Physical Chemistry C 2013, 117, 25360.

[17] H. W. Liu, D. Y. Chang, W. Y. Chiu, S. P. Rwei, L. Wang, Journal of Materials Chemistry 2012, 22, 15586.

[18] F. Steiner, S. Foster, A. Losquin, J. Labram, T. D. Anthopoulos, J. M. Frost, J. Nelson, Materials Horizons 2015, 2, 113.

[19] W. Shi, X. Hou, T. Liu, X. Zhao, A. B. Sieval, J. C. Hummelen, T. J. S. Dennis, Chemical Communications 2017, 53, 975.

[20] T. Liu, I. Abrahams, T. J. S. Dennis, Journal of Physical Chemistry A 2018, 122, 4138.

[21] F. Zhang, W. Shi, J. Luo, N. Pellet, C. Yi, X. Li, X. Zhao, T. J. S. Dennis, X. Li, S. Wang, Y. Xiao, S. M. Zakeeruddin, D. Bi, M. Grätzel, Advanced Materials 2017, 29, 1606806.

[22] T. Umeyama, H. Imahori, Accounts of Chemical Research 2019, 52, 2046.

[23] J. M. Fernández-Garciá, P. J. Evans, S. Filippone, M. Á. Herranz, N. Martín, Accounts of Chemical Research 2019, 52, 1565.

[24] J. R. Brandt, F. Salerno, M. J. Fuchter, Nature Reviews Chemistry 2017, 1, 1.

[25] G. Yang, Y. Si, Z. Su, Journal of Physical Chemistry A 2011, 115, 13356.

[26] P. Josse, L. Favereau, C. Shen, S. Dabos-Seignon, P. Blanchard, C. Cabanetos, J. Crassous, Chemistry - A European Journal 2017, 23, 6277. 
[27] K. Manoli, M. Magliulo, L. Torsi, Springer, Cham, 2013, pp. 133-176.

[28] F. Zinna, M. Pasini, F. Galeotti, C. Botta, L. di Bari, U. Giovanella, Advanced Functional Materials 2017, 27, 1603719.

[29] O. ben Dor, S. Yochelis, S. P. Mathew, R. Naaman, Y. Paltiel, Nature Communications 2013, $4,1$.

[30] X. Zhang, J. Yin, J. Yoon, Chemical Reviews 2014, 114, 4918.

[31] L. Wan, J. Wade, F. Salerno, O. Arteaga, B. Laidlaw, X. Wang, T. Penfold, M. J. Fuchter, A. J. Campbell, ACS Nano 2019, 13, 8099.

[32] J. R. Brandt, X. Wang, Y. Yang, A. J. Campbell, M. J. Fuchter, Journal of the American Chemical Society 2016, 138, 9743.

[33] B. Kunnen, C. Macdonald, A. Doronin, S. Jacques, M. Eccles, I. Meglinski, Journal of Biophotonics 2015, 8, 317.

[34] R. Farshchi, M. Ramsteiner, J. Herfort, A. Tahraoui, H. T. Grahn, Applied Physics Letters 2011, 98, 162508.

[35] J. Gilot, R. Abbel, G. Lakhwani, E. W. Meijer, A. P. H. J. Schenning, S. C. J. Meskers, Advanced Materials 2010, 22, E131.

[36] Y. Yang, R. C. da Costa, M. J. Fuchter, A. J. Campbell, Nature Photonics 2013, 7, 634.

[37] M. Schulz, F. Balzer, D. Scheunemann, O. Arteaga, A. Lützen, S. C. J. Meskers, M. Schiek, Advanced Functional Materials 2019, 29, 1900684.

[38] L. Wang, Y. Xue, M. Cui, Y. Huang, H. Xu, C. Qin, J. Yang, H. Dai, M. Yuan, Angewandte Chemie 2020, 132, 6504.

[39] J. Peng, B. P. Cumming, M. Gu, Optics Letters 2019, 44, 2998.

[40] C. Chen, L. Gao, W. Gao, C. Ge, X. Du, Z. Li, Y. Yang, G. Niu, J. Tang, Nature Communications 2019, 10, 1.

[41] N. Y. Kim, J. Kyhm, H. Han, S. J. Kim, J. Ahn, D. K. Hwang, H. W. Jang, B.-K. Ju, J. A. Lim, Advanced Functional Materials 2019, 29, 1808668.

[42] W. Li, Z. J. Coppens, L. v. Besteiro, W. Wang, A. O. Govorov, J. Valentine, Nature Communications 2015, 6, 1 .

[43] F. Djojo, A. Herzog, I. Lamparth, F. Hampel, A. Hirsch, Chemistry - A European Journal 1996, 2, 1537.

[44] A. Hirsch, I. Lamparth, H. R. Karfunkel, Angewandte Chemie International Edition in English 1994, 33, 437.

[45] T. Cao, N. Chen, G. Liu, Y. Wan, J. D. Perea, Y. Xia, Z. Wang, B. Song, N. Li, X. Li, Y. Zhou, C. J. Brabec, Y. Li, Journal of Materials Chemistry A 2017, 5, 10206.

[46] C. Thilgen, F. Diederich, Chemical Reviews 2006, 106, 5049.

[47] H. Ito, Y. Ishida, K. Saigo, Tetrahedron Letters 2005, 46, 8757.

[48] H. Goto, N. Harada, J. Crassous, F. Diederich, Journal of the Chemical Society. Perkin Transactions 2 1998, 1719.

[49] G. Pescitelli, T. Bruhn, Chirality 2016, 28, 466.

[50] F. Djojo, A. Hirsch, Chemistry - A European Journal 1998, 4, 344.

[51] H. Tanaka, Y. Inoue, T. Mori, ChemPhotoChem 2018, 2, 386.

[52] F. Plasser, Journal of Chemical Physics 2020, 152, 084108.

[53] R. L. Martin, Journal of Chemical Physics 2003, 118, 4775.

[54] J. A. Schellman, Chemical Reviews 1975, 75, 323. 
$E$

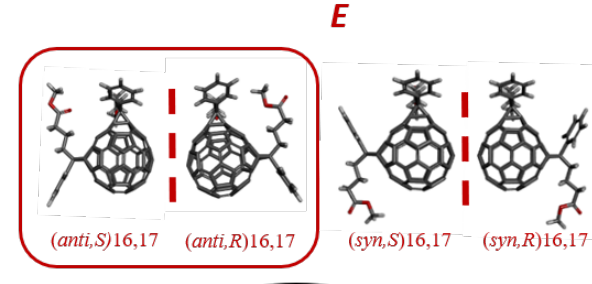

Cis-3

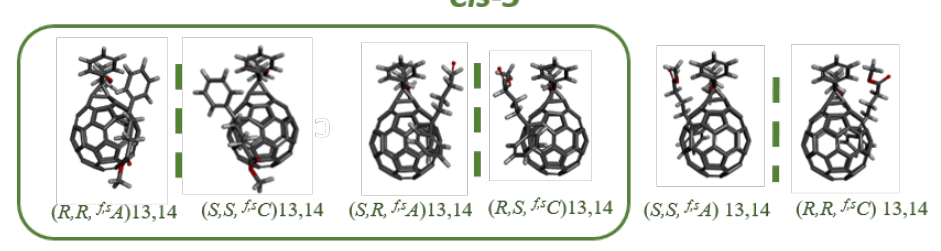

Trans-2

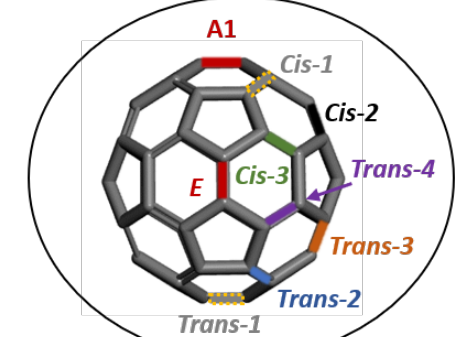

Cis-2
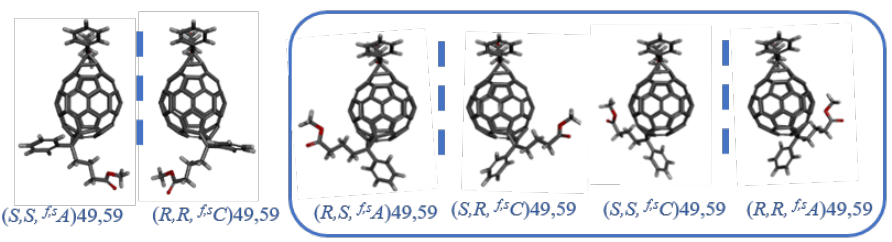

Trans-3
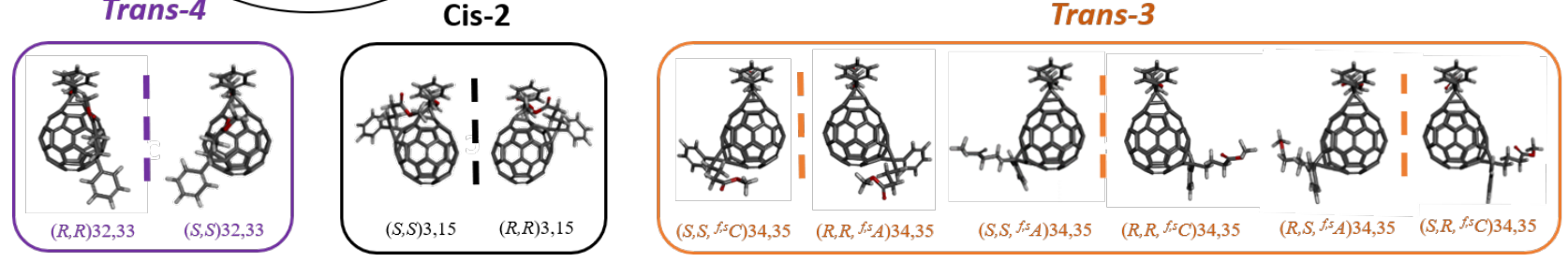

Figure 1. The 13 pairs of Bis[60]PCBM enantiomers, categorised into six bond types according to the positioning of the second adduct relative to 1,9 position of the first addend (A1): Cis-2 (black), Cis-3 (green), E (red), Trans-2 (blue), Trans3 (orange) and Trans-4 (purple) with dotted mirror planes depicted. For each isomer, $R / S$ descriptors refer to addend stereochemistry and ${ }^{f, s} C^{f f s} A$ to the fullerene stereochemistry using the method defined by Diederich and Thilgen ${ }^{[46]}$ All isomer labels should be proceeded by 'bis[60]PCBM', i.e. $\left(S, S,{ }^{f, s} C\right) 13,14$ becomes $\left(S, S,{ }^{f, s} C\right) 13,14-$ bis[60]PCBM. The enantiomeric pairs contained within boxes represent those isolated in this study. The stereochemical nomenclature system is further described in the SI. Files with rotatable structures for each enantiomer and larger images with more viewpoints are included as SI. 
a)

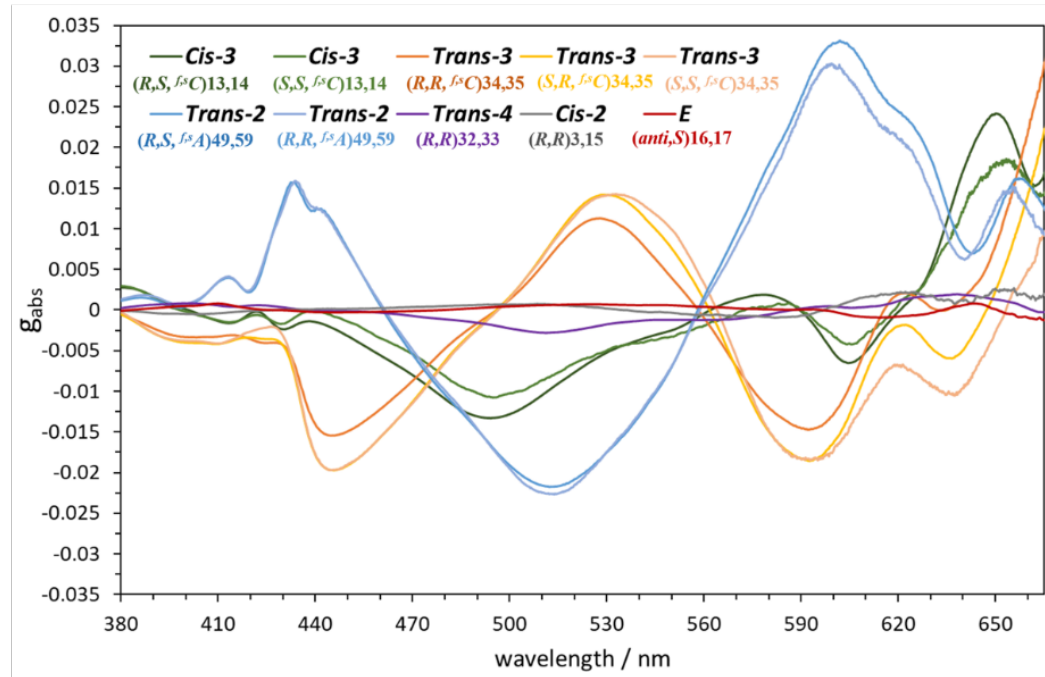

b)
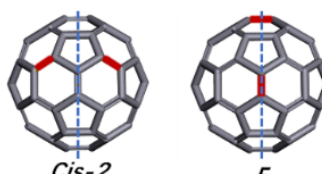

E

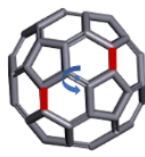

Cis-3

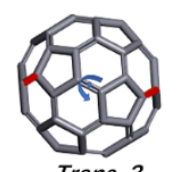

Trans-3

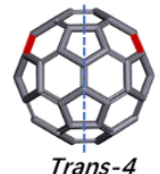

Trans-4

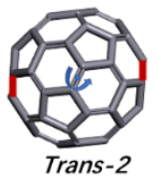

c)

(anti,S)16,17-bis[60]PCBM

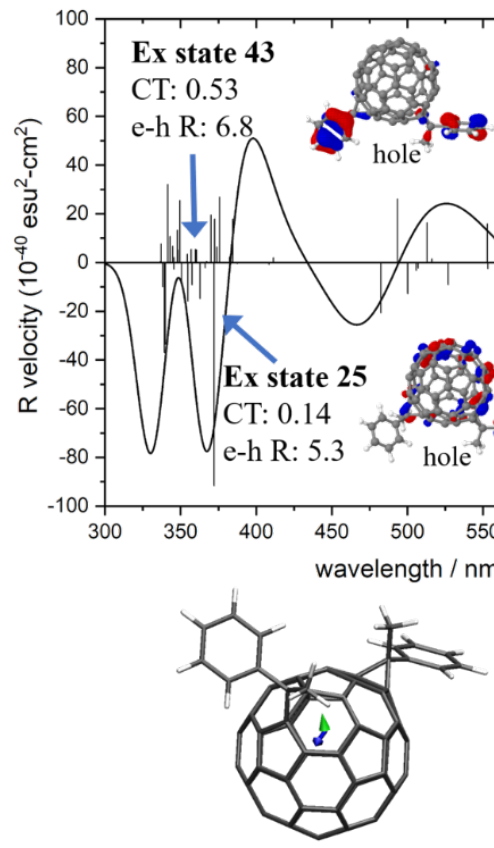

$\left(S, S,{ }^{\mathrm{f}, \mathrm{s}} C\right) 34,35$-bis[60]PCBM
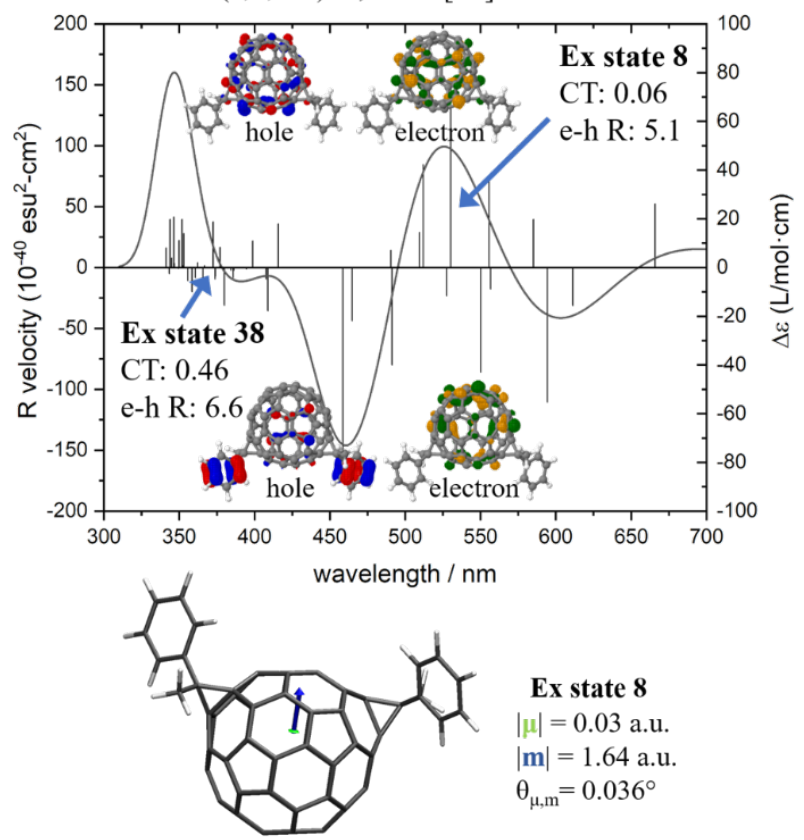

Ex state 8

$|\mu|=0.03$ a.u.

$|\mathrm{m}|=1.64$ a.u.

$\theta_{\mu \mathrm{m}}=0.036^{\circ}$

$|\mathrm{m}|=1.05$ a.u
$\theta_{\mu \mathrm{m}}=148.12^{\circ}$

Figure 2. a) Absorption dissymmetry factor $g_{\text {abs }}$ of one enantiomer per pair of all the isolated bis[60]PCBM enantiomers

from $380 \mathrm{~nm}$ to $700 \mathrm{~nm}$. b) Six addition patterns on the cage (two types): non-inherently chiral with mirror symmetry

(upper row) and inherently chiral with $\mathrm{C}_{2}$ symmetry (bottom row). c) Calculated CD spectra of the $E$ isomer $($ anti,S)16,17

(left) and the Trans-3 isomer $\left(S, S,{ }^{f, s} C\right) 34,35$ (right). The insets highlight the states with highest rotatory strength (state

25 and state 8 ) and the states with highest charge transfer character (state 43 and state 38 ). CT refers to the degree of charge transfer character and e-h R indicates the Root Mean Square distance of the electron-hole pair. The molecular pictures represent the distribution of the pair of "hole" (where the electron leaves upon excitation) and "electron" (where 
the excited electron goes to) for that excited state. On the bottom, electric and magnetic transition dipoles for the excited state 25 and 8 are displayed.

a)

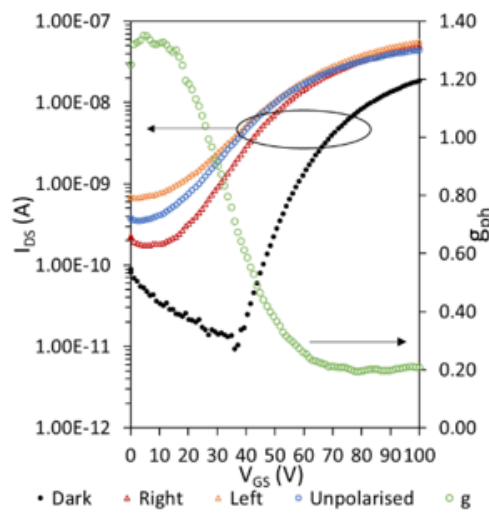

d)

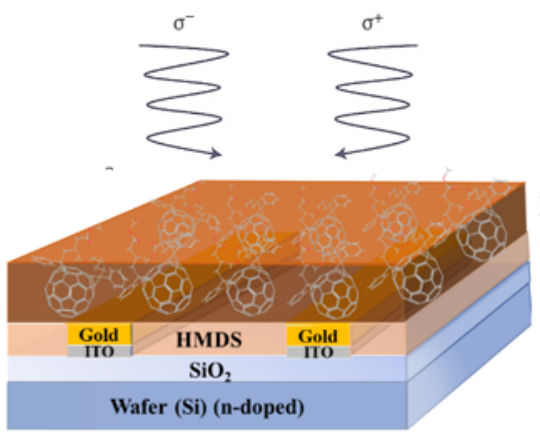

b)

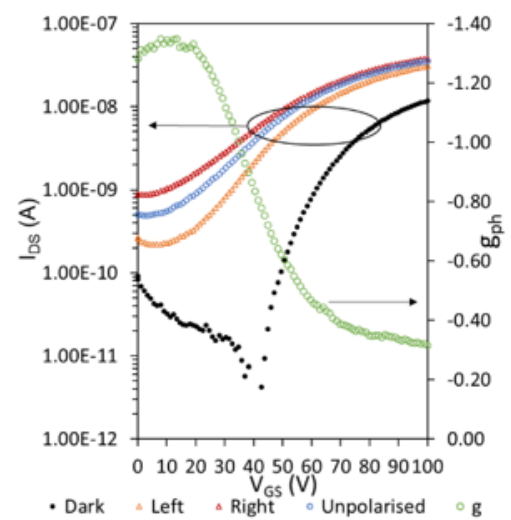

e)

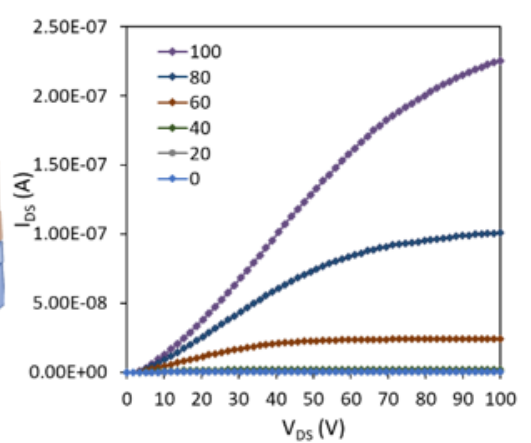

c)

f)
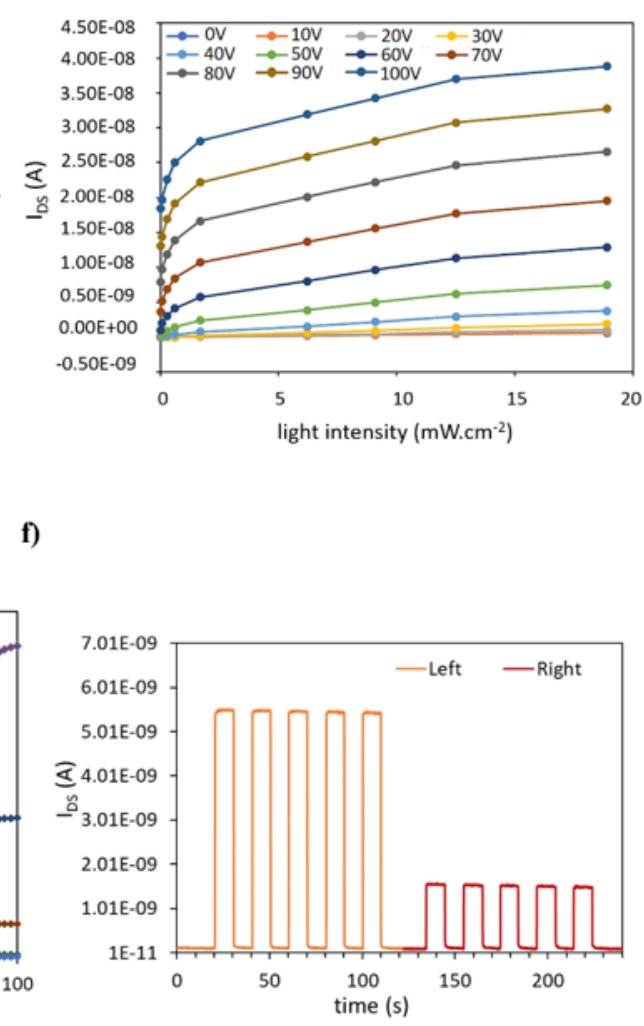

Figure 3. Device characteristics of Bis[60]PCBM enantiomer pure photo-FETs. Variation of transfer curves of (anti,S)16,17-bis[60]PCBM (a) and (anti,R)16,17-bis[60]PCBM (b) upon exposure to unpolarised, left-handed and righthanded CP light with $12 \mathrm{~mW} \mathrm{~cm}^{-2}$, compared to curves in the dark. $V_{\mathrm{Ds}}=20 \mathrm{~V}$. Dissymmetry factors for the photocurrent generation ( $\left.\mathrm{g}_{\mathrm{ph}}\right)$ was extracted and shown using green dots. c) The light intensity response of the photoFET from 0.06 $\mathrm{mW} \mathrm{cm}{ }^{-2}$ to $18.9 \mathrm{~mW} \mathrm{~cm}^{-2}$ at different gate voltage from $0 \mathrm{~V}$ to $100 \mathrm{~V}, V_{\mathrm{DS}}=20 \mathrm{~V}$. d) The device structure of photoFETs. e) Output characteristics of (anti,S)16,17-bis[60]PCBM photoFET at gate voltage between 0 and $100 \mathrm{~V}$. f) Time-resolved left- and right-handed CPL photoresponse was measured at $405 \mathrm{~nm}$ with an intensity of $11.6 \mathrm{~mW} \mathrm{~cm}{ }^{-2}$, a frequency of $0.05 \mathrm{~Hz}$ and $V_{\mathrm{DS}}=V_{\mathrm{GS}}=40 \mathrm{~V}$, showing a high stability and reversibility. 